\title{
El Método de los Valores Tipológicos (MVT). Ideas para un método de identificación y de prevaloración cualitativa de áreas a urbanizar ${ }^{1}$
}

\author{
Rodrigo Vidal Rojas ${ }^{2}$
}

\begin{abstract}
RESUMEN
Los estudios de urbanistas, geógrafos, economistas y sociólogos, como también la experiencia acumulada de los promotores inmobiliarios y de los tasadores de propiedades, permiten identificar y construir una importante cantidad de datos, respecto de predios urbanos y de predios urbanizables. Durante la investigación que funda este texto, fueron analizados una importante cantidad de esos datos, lo que nos permitió construir parámetros que expresan cualidades específicas del suelo urbano: orientación, nivel de seguridad, accesibilidad, etc. En total, se elaboraron 22 parámetros. Este texto propone un método de tratamiento de esos parámetros, con la finalidad de obtener una prevaloración cualitativa de futuros predios a urbanizar. Esta prevaloración puede constituir una importante herramienta prospectiva de planificación de las inversiones en suelo urbanizable por parte del Estado.
\end{abstract}

Palabras clave: Suelo urbano, método de valoración, parámetros de urbanización.

\begin{abstract}
The studies of city planners, geographers, economists and sociologists, including the comprehensive experience of real estate promoters, bring us to hand an important and relevant amount of data; respect the urban land market with their availabilities. The research that supports this paper has had the opportunity to analyze all this available data. This permitted us to identify parameters that express qualities values for this urban land availability: like orientation, level of security, accessibility and others. Altogether, we are considering 22 parameters. This paper proposes a methodology of analysis of those parameters with the purpose of obtaining future urban land availability. This validation will constitute an important urban growth prospective tool for state investment planning.
\end{abstract}

Key words: Urban land market, methodology, parameters of urbanization.

1 Artículo recibido el 11 de abril de 2007 y aceptado el 2 de enero de 2008. Este es el tercer artículo que surge de la investigación titulada Modelación computacional del impacto del poblamiento de las zonas periurbanas por grupos de ingreso medio-alto sobre el valor y el destino del suelo de reserva en el Gran Santiago, que se desarrolló con financiamiento de la
Dirección de Investigación Científica y Tecnológica (DICYT) de la Universidad de Santiago de Chile.

2 Arquitecto, Máster en Ciencias Sociales, Máster en Diseño Urbano y Ordenamiento Territorial, Doctor en Geografía Urbana, profesor e investigador Escuela de Arquitectura, Universidad de Santiago de Chile (Chile). E-mail: rvidal@usach.cl 
La expansión física es una característica innata de la ciudad (Geddes, 1915). Esta expansión responde históricamente a la acción del Estado y de las municipalidades para proveer vivienda social y desarrollar la industria en suelos de bajo precio, pero de manera central es una consecuencia de las acciones de inversión por parte de los promotores inmobiliarios privados. Esta política de desarrollo periurbano aparece bien fundada sobre varios fenómenos complementarios que adquieren toda su fuerza a partir de la segunda mitad del siglo XX en Europa $y$ durante los últimos 25 años en Chile: los precios relativamente bajos del suelo, la disponibilidad de suelo, el desarrollo del transporte individual, entre otros (Bigot, 1994: 51). Esta expansión se produce siguiendo invariablemente los mismos patrones que provocan siempre los mismos efectos: disminución de suelo agrícola, encarecimiento de los costos y tiempos de transporte, agravamiento de la crisis ambiental producto del aumento del parque automotriz y de las distancias de recorrido, construcción de nuevas vías financiadas en parte por un gran número de ciudadanos contribuyentes que no se benefician con su construcción, entre otros.

Martín Lou y Múscar subrayan un tercer factor de crecimiento urbano en América del Sur a partir de los años ochenta: "Dada la escasez de recursos públicos para la construcción y el mantenimiento de la ciudad, los sectores populares han asumido la construcción de áreas cada vez más extensas" (1992: 258259), áreas disponibles preferentemente en la periferia de la ciudad. Este crecimiento de los espacios periféricos es, en términos generales, producto del modelo económico, de la diversificación de funciones de la ciudad, del crecimiento demográfico, de las nuevas tecnologías, entre otros, como lo revela Jacqueline Beaujeu-Garnier (1997: 77-90).

En Europa, algunas prácticas de diversos orígenes han contribuido a disminuir el ritmo de expansión que experimentaron las ciudades desde la época de la industrialización, fomentando el desarrollo de ciudades pequeñas e intermedias y orientando las políticas urbanas hacia un mayor equilibrio territorial del espacio urbano, redefiniendo la ciudad de fines del siglo XX y comienzos del siglo XXI (Remy et Voyé, 1992).
Algunas de estas prácticas son los procesos de gentrification (reciclaje de áreas y edificios históricos), de new urbanism (en lo que respecta la reconquista de sectores ya urbanizados) y de renovación urbana (en la ocupación residencial de antiguas zonas industriales) con el traslado de la industria fuera de los márgenes del área urbanizada, acompañados de una verdadera voluntad de limitar la expansión física, basados en las nuevas corrientes de diseño y planificación urbana que se observan en Europa (Ascher, 2004: 55-85).

Por su parte, las grandes ciudades latinoamericanas se expanden, se recentralizan, siguen generando una fuerte presión centrífuga. El territorio se sigue desequilibrando y la macrocefalia capitalina caracteriza el ordenamiento urbano, con los efectos ya conocidos. En Chile, el respeto ciego a la iniciativa privada, consagrado en la Constitución Política de la República; una legalización de estos procesos desprovista de mecanismos reales de fiscalización en terreno; la creencia de las autoridades en que no hay que aplicar una camisa de fuerza a la ciudad a través del límite urbano; una memoria corta que olvida las consecuencias territoriales provocadas por la eliminación del límite urbano (19791984) y que olvida la urbanización en parcelas de agrado desarrollada en terrenos de $5.000 \mathrm{~m}^{2}$ creados con fines agrícolas; la incongruencia de haber aplicado un Programa de repoblamiento del centro de la ciudad sin prosecución a través de un proceso serio y planificado de densificación del vasto pericentro urbano (en el caso de Santiago) y el rol del propio Ministerio de Vivienda y Urbanismo que ha estimulado históricamente la expansión de la ciudad más allá del límite urbano, a través de los programas de subsidios habitacionales, explican en parte esta insistencia por expandir la ciudad.

Lamentar a posteriori los efectos de estos errores recurrentes resulta estéril. Con el fin de contribuir a un debate amplio y propositivo acerca de cómo aprovechar al máximo la experiencia acumulada en materia de desarrollo urbano, y lograr al mismo tiempo disminuir el impacto de la expansión urbana, se propone poner sobre la mesa de discusión el Método de los Valores Tipológicos (MVT). Primeramente, se exponen de manera sucinta la 
diversidad de factores que inciden en la formación del precio del suelo. En segundo lugar, se explica detalladamente el método. Antes de concluir se dan algunas orientaciones prácticas de aplicabilidad del método MVT.

Específicamente, en relación con el método de valores tipológicos se presentan las variables de base a considerar; se identifican y describen los parámetros generales de valoración cualitativa del suelo, detallando el procedimiento para la construcción del valor por unidad de suelo e ilustrando el método a través del estudio de trazados alternativos del territorio.

\section{La diversidad de factores que inciden en la formación del precio del suelo}

La formación de los precios del suelo en el medio urbano depende de factores que son no solo numerosos, sino además muy diversos: considera desde la calidad del subsuelo para un determinado uso, hasta el prestigio del barrio en el que se encuentra, incluido su potencialidad de uso futuro. Algunos de los factores que pueden determinar y modificar en el mediano y largo plazo el precio del suelo son: los cambios en los instrumentos de planificación territorial, la modificación en la estructura socioeconómica de la población, las variaciones de los niveles de seguridad urbana, las nuevas valoraciones sociales de un sector de la ciudad o la construcción cercana de una carretera o de un centro de servicios, entre otros.

Por lo demás, el precio del suelo, considerado como un dato económico de transacción, depende de la valoración social que ese suelo obtenga. Esa valoración es tributaria a su vez de la diversidad de factores antes subrayada, como también de la valoración del suelo en el juego de la oferta y de la demanda en el mercado. La existencia de potenciales compradores le otorga al suelo la calidad de recurso transable, por lo tanto valorable. Es a partir de ese momento que entran en juego los factores que permitirán la fijación de un precio de transacción.

En consecuencia, la valoración social del suelo antecede la fijación económica del precio. La literatura anglosajona y francófona $y$, en menor medida, la literatura hispana ofrece una gran cantidad de modelos de valoración del suelo urbano. En general, esos modelos se refieren a las características particulares del mercado territorial, a los componentes del precio del suelo y a los factores de variación de los precios.

Según Derycke (1987: 126-139), entre los modelos clásicos americanos destacan el modelo de Wingo y el modelo de William Alonso, de carácter más general que el anterior. Entre los modelos clásicos franceses se destacan el modelo de Gérard Maarek y el modelo de René Mayer. Ambos intentan perfeccionar los modelos americanos. "Todos estos modelos tienen en común el esfuerzo por vincular el precio de los terrenos a los costes de transporte, además de integrar los mecanismos de formación de los valores del suelo a la teoría del crecimiento urbano" (Derycke, 1987: 127).

Granelle (1967: 52) destaca que el mercado del suelo presenta cuatro características que contribuyen a modificar el precio. Estas son las siguientes: (1) es un mercado de imperfección en la competencia, (2) es un mercado de especulación, (3) es un mercado complementario del mercado de la construcción y, producto de las anteriores, (4) es un mercado fuertemente orientado al alza. Además, los autores franceses coinciden en que el valor agrícola del suelo; los costos de ordenación del suelo; las rentas de escasez (plusvalías por la demora de puesta en situación urbanizada) y de situación (todas las particularidades del emplazamiento); y la accesibilidad del terreno, son componentes esenciales del precio final. A su vez, Derycke (1987: 119) insiste en la existencia de tres factores a su juicio preponderantes en la variación y formación del precio: "la distancia al centro; la red de transporte y el crecimiento urbano".

\section{El Método de los Valores Tipológicos}

Conviene advertir que el modelo propuesto se orienta evaluar la percepción de calidad de áreas urbanizables, a partir de parámetros seleccionados, para una mayor viabilidad económica de la urbanización, localizando 
en el espacio los ámbitos periurbanos con mayor probabilidad de demanda de urbanización. Esto permite acercar las expectativas de valor que un usuario posee en relación con el precio ofertado del suelo, al grado de satisfacción de ese usuario, una vez el suelo urbanizado y ocupado. Sin duda alguna, este método se complementa con otros que evalúen, por ejemplo, la viabilidad ecológica y ambiental de la urbanización.

A través de este modelo se intenta contribuir a una urbanización que se aproxime de mejor forma a las características de la demanda del suelo periurbano. Este estudio no ha tenido por objeto estudiar los parámetros que justificarían la no urbanización del suelo, producto de valores patrimoniales, arqueológicos, ecológicos, paisajísticos u otros. Tampoco contempla la oportunidad de la urbanización en términos de ritmo, programación, velocidad de los diferentes sectores a urbanizar. No es un método para la toma de decisión de la urbanización, ni para la determinación de la forma de esa urbanización, pero sí contribuye con datos relevantes a esa toma de decisión.

El Método de los Valores Tipológicos (MVT) es un método para la valoración económica y cualitativa de un área a urbanizar a partir de parámetros geográficos, sociales, económicos, físico-ambientales del entorno, paisajísticos, de accesibilidad, de la estructura del suelo y de la forma del territorio.

La principal fortaleza del modelo está en permitir conocer con anticipación las orientaciones del mercado y los mecanismos que permiten orientar la forma del crecimiento y la organización del territorio. Consiste básicamente en aplicar una tabla de valores relativos de los diversos parámetros que intervienen en la percepción social del suelo periurbano para prever los efectos de plusvalía de un terreno en función de diversos escenarios de urbanización.

Esta percepción social del territorio condiciona en mayor o menor grado el interés que el suelo provoca en el usuario e incide directamente en el precio del mismo en función de la mayor o menor demanda que surge. Esto se debe a que el precio del suelo urbano es un producto directo del juego de la oferta y de la demanda, fuertemente condicionado por dos factores esenciales: el prestigio y la moda. El primero, más permanente, tiene que ver con la valoración social del suelo y el segundo, más variable, con ciertos factores estacionales que ponen en valor cualidades específicas en un momento histórico dado.

La principal implicancia de lo anterior es que el mayor o menor precio de un predio no refleja proporcionalmente su mayor o menor calidad objetiva. Por calidad objetiva se entienden aquellos parámetros que permiten establecer inequívocamente cualidades que serán reconocidas por todos en función de una finalidad: así por ejemplo, para el promotor inmobiliario, la accesibilidad, el grado de consolidación del suelo y la proximidad relativa a los equipamientos serán valores a considerar. Para un romántico, la calidad del paisaje será tan importante como para el agricultor saber de qué calidad es el suelo. Al no existir cualidades únicas para finalidades convergentes, es necesario ponderar su incidencia en el valor final.

Es lo que Ilamamos una valoración social de los predios urbanos y que no siempre es considerada en los proyectos inmobiliarios, por ser fundamentalmente cualitativa y difícil de cuantificar con las herramientas actuales. Muchos terrenos no se venden porque su precio se eleva a niveles incongruentes con la calidad del lugar. Esto lleva a generar una oferta inmobiliaria que en determinados casos supera la demanda lo que se explica en parte por el hecho de que la valoración del suelo no es sinónimo de encarecimiento. El prestigio y la moda aumentan la demanda, encarecen la oferta y disminuyen el mercado disponible, generando al mismo tiempo una fuerte presión sobre suelos no urbanizados y de creciente valoración social y cultural. Sin embargo, la mayor demanda muchas veces tiende a saturar el mercado en desmedro de aquello que le dio su prestigio y lo puso de moda. La devaluación cualitativa del suelo acompaña su encarecimiento, transformando grandes áreas de la ciudad en zonas caras, de prestigio, de imagen positiva pero de una cada vez menor calidad de vida. Dos ejemplos claros de este proceso son el Centro Histórico de Santiago y su permanente degradación cualitativa desde los años 50 y la comuna de Providencia, en especial las áreas aledañas al eje Providencia-11 
de Septiembre y a la calle Bilbao, como consecuencia de la expansión de la ciudad al nororiente, lo que transforma esta comuna en una comuna de tránsito. En ambos casos, el alto tráfico vehicular (privado y colectivo) con sus corolarios de congestión, ruido, contaminación del aire y visual, como también la presencia muchas veces agresiva del comercio y los servicios, hacen de estas áreas lugares cada vez menos habitables. No obstante, sobre todo en el segundo caso, los precios mantienen índices elevados.

Por otro lado, los loteos y urbanizaciones en comunas con importantes áreas no urbanizadas, se desarrollan muchas veces sobre la base de presunciones y en muchas ocasiones con éxito de ventas. El problema es que con el tiempo, la decepción de los habitantes revela el abismo entre expectativas (en relación al precio) y beneficios (en relación a la calidad de vida real obtenida).

El objetivo central del Método de los Valores Tipológicos es integrar la valoración social del suelo en las decisiones de urbanización, tanto en la estrategia de localización y emplazamiento como en la organización y diseño del territorio. Es un método más bien apriorístico que mantiene el precio del suelo dentro de la lógica de la oferta y de la demanda, pero que interviene indirectamente en la construcción del precio en la medida en que otorga una mayor información al demandante. Esto se debe a que a pesar de su fuerte dependencia del libre mercado "la cuestión inmobiliaria sigue siendo un tema político difícil y diseminado de resquicios, comportando aspectos de lucha entre grupos de interés, como también aspectos de ética social" (Bridel, 1996: 61).

\section{Las variables del Método de los Valores Tipológicos}

De lo anterior resulta evidente la necesidad de establecer una distinción entre precio y valor del suelo. "El precio relativo de un bien o servicio está determinado, según los economistas clásicos, por la cantidad de recursos naturales y de trabajo necesario para su producción, lo que constituye un indicador de su valor. Sin embargo, es necesario intervenir, en particular para el bien tierra, las relaciones de poder o de dependen- cia, especialmente a través de la propiedad. El propietario detenta un derecho -algunos dirán un privilegio- el cual le da, en conformidad con la reglas de nuestra sociedad liberal, la capacidad de cobrarlo, a condición que exista una demanda" (Bridel, 1996: 69). Lo anterior permite a Bridel establecer que el precio inmobiliario y del suelo se constituye en función de a lo menos tres factores esenciales:

1. Los factores generales (legislación, nivel global de precios, escasez del suelo, etc.);

2. Los factores de barrio o de área (accesibilidad, calidad ambiental, nivel social, disposiciones de la ordenanza, etc.); y

3. Los factores de la parcela (tamaño, topografía, cualidades geomorfológicas, acceso a los equipamientos y a los transportes públicos, etc.).

A su vez, estos factores dependen de variables socioculturales, entre las cuales cabe destacar: la diversidad de intereses en torno al suelo, la alta necesidad de endeudamiento hipotecario para la adquisición de terrenos y las facilidades de acceso al crédito, las tendencias de legislación vigente, el prestigio y la moda antes indicados, entre otros.

Lo anterior explica la alta vulnerabilidad del precio del suelo cuando este se caracteriza por su inflación, vulnerabilidad que perjudica al promotor e inversionista. Es por ello que la tendencia a la estabilidad de los precios relativos, la cual conduce a una baja en la tasa de ganancia de las empresas inmobiliarias, incide directamente en la tendencia expansionista del mercado inmobiliario, cuyo objetivo es la deslocalización del alza del precio del suelo, a través de la reinversión de las ganancia en suelos de mayor plusvalía, con el coincidente aumento del precio (por los valores incorporados), sin que ello se traduzca en inflación respecto del mercado global. De esta manera, el inversionista incorpora el precio del suelo en el precio global de la inversión inmobiliaria (el que se traduce en el precio de venta del inmueble) la que incorpora además factores indirectos a la inversión (vistas, paisaje, aire, accesibilidad) además de los factores directos (calidad del inmueble). Al mismo tiempo esta deslocalización se transforma en 
una herramienta de negociación del oferente, respecto del demandante, lo que permite aumentar el precio.

Lo anterior es directamente concordante con las dos primeras de las cuatro conclusiones para Santiago propuestas por Sabatini, a saber: "El precio del suelo es dependiente del precio de venta de las viviendas, y no al revés" y "El precio del suelo está influido por relaciones de fuerza entre promotores y propietarios" (Sabatini, 1990: 65). Para comprender esta relación de fuerza, e intervenir en ella, es importante identificar las variables independientes y dependientes implícitas en el juego de la oferta y la demanda, las que pueden ser observadas mediante parámetros bien precisos. Llamaremos variables del tipo dependiente a aquellas que están directamente condicionadas a partir de los tres factores identificados por Bridel y podrían ser objetivadas. Las variables independientes son aquellas propias del lugar a intervenir y cuya valoración depende de factores culturales y de imagen, fundamentalmente subjetivas. En el título siguiente nos interesa reconocer los parámetros que emergen de esas variables y que permiten valorar el suelo más que especificar la diferencia entre los dos tipos de variables.

Más arriba se explica que el MVT consiste básicamente en aplicar una tabla de valores relativos de los diversos parámetros que intervienen en la percepción social del suelo periurbano para prever los efectos de plusvalía de un terreno en función de diversos escenarios de urbanización. Se trata de un método del tipo lógico-deductivo, en la medida en que su punto de partida es una determinación de valores generales relativos y ponderados, no dependiente de un lugar o lugares específicos, para luego, a través de acercamientos sucesivos, ser aplicado en lugares seleccionados. Su carácter lógico es una aplicación del pensamiento filosófico de Emmanuel Kant, fundador de la lógica trascendental o material, quien considera la lógica como el estudio del pensamiento aplicado a la realidad. Esta definición se acerca a la idea moderna según la cual la lógica es el arte de conducir adecuadamente su razón en la búsqueda del conocimiento de las cosas, tanto para instruirse uno mismo, como para instruir a los demás. El método lógico-deductivo aplicado es entonces una estrategia que permite llegar de conceptualizaciones generales a aplicaciones reales, a través de la construcción de un pensamiento orientado a crear conocimiento.

Las etapas sucesivas de este método lógico-deductivo son cinco:

1. Identificación y descripción de los parámetros generales de valoración cualitativa del suelo;

2. Construcción del valor por unidad de suelo;

3. Estudio de trazados alternativos del territorio;

4. Aplicación de la tabla valórica-cualitativa al territorio; $y$

5. Aplicaciones concretas.

\section{Identificación y descripción de los parámetros generales de valoración cualitativa del suelo}

La primera y fundamental etapa del MVT consiste en identificar aquellos parámetros recurrentes al momento de elegir un terreno a urbanizar y que constituyen el fundamento sobre el cual se construye el valor del mismo. Para identificar estos parámetros utilizamos cuatro herramientas:

1. Estudio de las cualidades ofrecidas en la oferta inmobiliaria de los alrededores de Santiago, según lo publicado en el diario El Mercurio, durante un período de un año;

2. Estudio de los parámetros considerados al momento de evaluar propiedades por parte de tasadores profesionales;

3. Estudio de la percepción de las razones evocadas por los compradores, según la información entregada por diversos profesionales de empresas inmobiliarias (empresarios, arquitectos, corredores de propiedades); y

4. Estudio de la bibliografía pertinente.

Los cuatro estudios anteriores revelaron los parámetros que a continuación se exponen, los que se definen sucintamente, y se ordenan según la prioridad acordada por los usuarios. Se trata de parámetros interdepen- 
dientes: por ejemplo, la percepción del nivel de seguridad estará en directa relación con el nivel socioeconómico del entorno, de la misma manera en que el costo de la edificación es tributario también del tipo de relieve.

Los parámetros, individualmente definidos son los siguientes:

- Parámetros de primera prioridad: se trata de aquellos parámetros que son considerados en primera instancia por los futuros usuarios, antes de la adquisición de un terreno. Las motivaciones de inversión pasan en gran medida por la consideración de estos parámetros de inversión o del inversionista.

- Parámetros de segunda prioridad: son aquellos parámetros que condicionan fundamentalmente los costos, la forma, la calidad de la urbanización, condiciones que, en definitiva, afectarán los costos de construcción de los edificios. Son los parámetros de urbanización o del urbanista.

- Parámetros de tercera prioridad: una vez decidida la inversión y la urbanización, corresponde determinar las condiciones generales de la arquitectura y de la construcción, considerando estos parámetros que actúan directamente sobre la decisión del proyecto a edificar. Son los parámetros de la construcción o del arquitecto.

\section{Parámetros de primera prioridad o de inversión}

Orientación: se refiere a la disposición y proporción del terreno respecto del asoleamiento. Una orientación norte significa que el terreno se orienta principalmente de sur a norte con acceso desde el norte. Una orientación sur significa que el terreno se orienta de norte a sur con acceso desde el sur.

Nivel socioeconómico del entorno: se refiere a las características socioeconómicas actuales y permanentes del entorno del área a urbanizar (nivel de ingresos, tipo de urbanización, seguridad, etc.).

Entorno socioeconómico del lugar: se refiere a las características socioeconómicas actuales y permanentes del área a urbanizar (nivel de ingresos, tipo de urbanización, seguridad, etc.). En otras palabras, el nivel económico y cultural de los nuevos residentes, la calidad de las construcciones, etc.

Presencia de ruidos desagradables: fundamentalmente referidos a circulación vehicular, actividades productivas, proximidad de aeropuertos, oportunidad de manifestaciones sociales, entre otros.

Distribución de unidades residenciales: se refiere al grado de independencia y distancia entre las unidades residenciales. A mayor distancia y privacidad, mayor valoración del suelo.

Nivel de seguridad: la seguridad garantizada contra la delincuencia en general $y$, en particular, a favor de la tranquila convivencia entre residentes, es un factor mayor de valoración. Esta valoración es tanto más importante cuanto menor son las oportunidades de tener que ejercer los instrumentos de seguridad disponible.

Propiedad de los terrenos disponibles: la absoluta legalidad de la posesión de los terrenos y la directa relación con los propietarios oferentes, sin mediación de terceros, es un factor bien evaluado al momento de valorar un terreno o área a urbanizar.

\section{Parámetros de segunda prioridad o de urbanización}

Tipo de paisaje: se refiere a las cualidades del paisaje concebido como las vistas posibles al entorno natural (río, montaña, valle, árboles, etc.). Esto también involucra las cualidades estéticas del área a urbanizar.

Accesibilidad a vías pavimentadas: es la proximidad, contigüidad o facilidad de acceder a vías pavimentadas.

Accesibilidad a fuentes de trabajo: es la proximidad a las fuentes de trabajo, o la disponibilidad de vías expeditas hacia las fuentes de trabajo (es el ejemplo de quienes se instalan en las proximidades de la Avenida Américo Vespucio por la mayor rapidez para acceder a sectores empresariales, al aeropuerto o a las intersecciones con las vías nacionales). 
Accesibilidad a servicios: es la proximidad a los centros de servicios, o la disponibilidad de vías expeditas hacia ellos (es el ejemplo de quienes se instalan en las proximidades de la Avenida Américo Vespucio, del eje Ruta 68-Apoquindo por la mayor rapidez para acceder a los centros de servicios: centro histórico, centro de negocios, etc.).

Accesibilidad a transporte público: es la proximidad a las vías de tránsito del transporte público (este aspecto es medianamente valorado en los grupos de ingreso medio y bajo, y tiene una menor valoración en los grupos de ingreso alto).

Relación al área urbana consolidada: cuando se trata de nuevas áreas residenciales, esta relación es requerida respecto de los centros de abastecimiento y servicios, ya sea que existan a proximidad (el caso de Huechuraba), ya sea que estén en proyecto de ser desarrollados en paralelo (el caso de los faldeos cordilleranos de La Florida y Peñalolén), ya sea que el proyecto inmobiliario los considere (el caso de algunos loteos de Chicureo).

Nivel de concentración humana: se refiere a la mayor o menor densidad de ocupación en las nuevas áreas a urbanizar.

Coeficiente de constructibilidad: referido aquí a la mayor oportunidad de ocupación del suelo asociada a una mayor oportunidad de superficie de edificación.

Tipo de seguridad: se refiere a si la seguridad será dada por Carabineros e Investigaciones, por guardias armados de empresas privadas, o por guardias sin armas de fuego.

Situación de los terrenos disponibles: se refiere a la cantidad de dueños y al grado de saneamiento de los terrenos.

Estructura de propiedad de los predios: se refiere al tipo de propietario (público, privado o mixto).

Estructura etárea de la población: se refiere a si la población se constituye prioritariamente de adultos mayores, familias con hijos en edad escolar o matrimonios y solteros jóvenes. Sin duda que esta percepción dependerá de la posición de cada cual en dicha estructura. La tendencia es a buscar vecinos pares.

\section{Parámetros de tercera prioridad o de construcción}

Tipo relieve: si se trata de terrenos en pendiente suave o fuerte, a lo que se incorpora también las cualidades de un terreno plano, liso o escarpado.

Geometría o proporción de los terrenos disponibles: la predominancia del fondo en relación al frente será menos valorada que la predominancia de un gran frente, con proporciones que tiendan al cuadrado.

Costo general de la edificación: se refiere al incremento del costo real de la edificación, provocado por las características del terreno: pendiente, tipo de suelo, nivel de la napa freática, etc.

El estudio de estos parámetros permitió confirmar que, por una parte, los aspectos más valorados por los compradores al momento de elegir un predio son: la orientación, el nivel socioeconómico del entorno, el entorno socioeconómico del lugar, la presencia o no de ruidos desagradables, la forma de la distribución de las unidades residenciales, el nivel de seguridad del lugar y el tipo de propiedad de los terrenos disponibles. Por otra parte, los aspectos menos considerados (o despreciables) son: el tipo de relieve (a pesar que en los estratos económicos bajos este aspecto cobra importancia), la geometría o proporción de los terrenos disponibles y la estructura de edades de la población.

\section{Construcción del valor por unidad de suelo}

Sobre la base de estos parámetros construimos la tabla de valoración y ponderación de los parámetros considerados (Cuadro $\left.N^{0} 1\right)$. En ella figuran, en una columna, los 22 parámetros seleccionados, con sus correspondientes indicadores. Los indicadores de los parámetros se ordenan en función de una escala de notas de 0 a 7 . 
Cuadro $\mathrm{N}^{\circ} 1$

TABLA DE VALORACIÓN Y PONDERACIÓN DE LOS PARÁMETROS CONSIDERADOS*

Diccionario Campos en Mapinfo

\begin{tabular}{|c|c|c|c|c|c|c|c|c|c|c|c|}
\hline & & & & uf & $\mathrm{m}^{2}$ & $\mathrm{uf} / \mathrm{m}^{2}$ & ep & en & ponderación & puntaje & acumulado \\
\hline 1 & Orientación & 0 & & & & & 4 & 0 & 0,085 & 7 & 0,6 \\
\hline 1.1 & Nororiente & & 7 & & & & 1 & 0 & & & \\
\hline 1.2 & Norte & & 6 & & & & 1 & 0 & & & \\
\hline 1.3 & Norponiente & & 5 & & & & 1 & 0 & & & \\
\hline 1.4 & Oriente & & 4 & & & & 1 & 0 & & & \\
\hline 1.5 & Suroriente & & 3 & & & & 0 & 0 & & & \\
\hline 1.6 & Poniente & & 2 & & & & 0 & 0 & & & \\
\hline 1.7 & Surponiente & & 1 & & & & 0 & 0 & & & \\
\hline 1.8 & Sur & & 0 & & & & 0 & 0 & & & \\
\hline 2 & Costo edificación según relieve & $\mathrm{Cr}$ & & & & & 4 & 0 & 0,034 & 7 & 0,24 \\
\hline 2.1 & Plano en cima & & 7 & & & & 1 & 0 & & & \\
\hline 2.2 & Plano en valle & & 5 & & & & 1 & 0 & & & \\
\hline 2.3 & Pendiente suave & & 3 & & & & 1 & 0 & & & \\
\hline 2.4 & Pendiente fuerte & & 1 & & & & 1 & 0 & & & \\
\hline 3 & Tipo de relieve & $\operatorname{tr}$ & & & & & 4 & 0 & 0,016 & 7 & 0,11 \\
\hline 3.1 & Plano en cima & & 7 & & & & 1 & 0 & & & \\
\hline 3.2 & Plano en valle & & 5 & & & & 1 & 0 & & & \\
\hline 3.3 & Pendiente suave & & 3 & & & & 1 & 0 & & & \\
\hline 3.4 & Pendiente fuerte & & 1 & & & & 1 & 0 & & & \\
\hline 4 & Tipo de paisaje & tp & & & & & 3 & 3 & 0,034 & 7 & 0,24 \\
\hline 4.1 & Natural abierto & & 7 & & & & 1 & 0 & & & \\
\hline 4.2 & Natural cerrado & & 6 & & & & 1 & 0 & & & \\
\hline 4.3 & Seminatural & & 5 & & & & 1 & 0 & & & \\
\hline 4.4 & Poblado & & 4 & & & & 0 & 1 & & & \\
\hline 4.5 & Urbano & & 3 & & & & 0 & 1 & & & \\
\hline 4.6 & Edificado & & 2 & & & & 0 & 1 & & & \\
\hline 5 & Accesibilidad a vías pavimentadas & avp & & & & & 1 & 1 & 0,034 & 7 & 0,24 \\
\hline 5.1 & Inmediata & & 7 & & & & 1 & 0 & & & \\
\hline 5.2 & Mediata & & 4 & & & & 0 & 0 & & & \\
\hline 5.3 & Lejana & & 1 & & & & 0 & 1 & & & \\
\hline 6 & Accesibilidad al trabajo & at & & & & & 1 & 1 & 0,034 & 7 & 0,24 \\
\hline 6.1 & Inmediata & & 7 & & & & 1 & 0 & & & \\
\hline 6.2 & Mediata & & 4 & & & & 0 & 0 & & & \\
\hline 6.3 & Lejana & & 1 & & & & 0 & 1 & & & \\
\hline 7 & Entorno socioeconómico del lugar & el & & & & & 1 & 1 & 0,085 & 7 & 0,6 \\
\hline 7.1 & Inmediata & & 7 & & & & 1 & 0 & & & \\
\hline 7.2 & Mediata & & 4 & & & & 0 & 0 & & & \\
\hline 7.3 & Lejana & & 1 & & & & 0 & 1 & & & \\
\hline 8 & Accesibilidad a servicios & as & & & & & 1 & 1 & 0,034 & 7 & 0,24 \\
\hline 8.1 & Inmediata & & 7 & & & & 1 & 0 & & & \\
\hline 8.2 & Mediata & & 4 & & & & 0 & 0 & & & \\
\hline 8.3 & Lejana & & 1 & & & & 0 & 1 & & & \\
\hline 9 & Accesibilidad al transporte público & atp & & & & & 1 & 1 & 0,034 & 7 & 0,24 \\
\hline 9.1 & Facilitada & & 7 & & & & 1 & 0 & & & \\
\hline 9.2 & No facilitada & & 1 & & & & 0 & 1 & & & \\
\hline 10 & Relación al área urbana consolidada & auc & & & & & 2 & 2 & 0,034 & 7 & 0,24 \\
\hline 10.1 & Central & & 7 & & & & 1 & 0 & & & \\
\hline 10.2 & Pericentral & & 5 & & & & 1 & 0 & & & \\
\hline 10.3 & Periférico & & 3 & & & & 0 & 0 & & & \\
\hline 10.4 & Periurbano & & 1 & & & & 0 & 1 & & & \\
\hline 10.5 & No urbano & & 0 & & & & 0 & 1 & & & \\
\hline 11 & Presencia de ruidos desagradables & rd & & & & & 6 & 6 & 0,085 & 7 & 0,6 \\
\hline 11.1 & Permanente & & 7 & & & & 2 & 2 & & 7 & \\
\hline 11.1.1 & Ausencia & & 5 & & & & 1 & 0 & & & \\
\hline 11.1.2 & Mínimo & & 3 & & & & 1 & 0 & & & \\
\hline 11.1.3 & tolerable & & 2 & & & & 0 & 0 & & & \\
\hline 11.1.4 & Molesto & & 1 & & & & 0 & 1 & & & \\
\hline 11.1 .5 & Dañino & & & & & & 0 & 1 & & & \\
\hline 11.2 & Esporádico & & & & & & 2 & 2 & & 7 & \\
\hline 11.2 .1 & Ausencia & & 7 & & & & 1 & 0 & & & \\
\hline 11.2 .2 & Mínimo & & 5 & & & & 1 & 0 & & & \\
\hline 11.2.3 & Tolerable & & 3 & & & & 0 & 0 & & & \\
\hline 11.2 .4 & Molesto & & 2 & & & & 0 & 1 & & & \\
\hline 11.2 .5 & Dañino & & 1 & & & & 0 & 1 & & & \\
\hline
\end{tabular}


Cuadro $\mathrm{N}^{\circ} 1$

(CONTINUACIÓN)

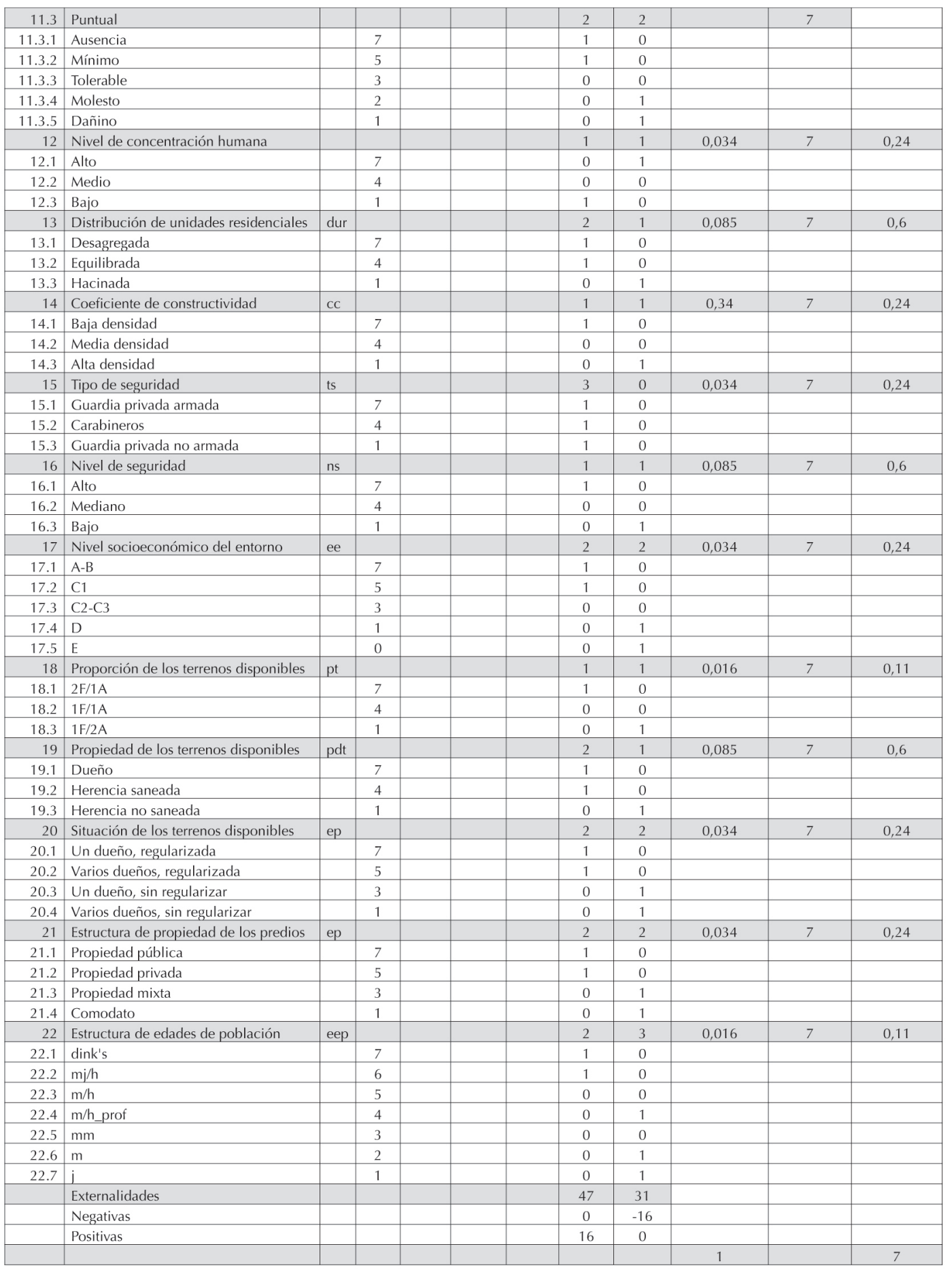

INVESTIGACIÓN DICYT - R. VIDAL, R. MARTIN, D. LAGOS, C. HERMOSILLA

Tabla de variables y parámetros que permiten construir la malla o manto de valoración del suelo periurbano

Santiago, 23 de octubre de 2000

Fuente: Elaboración propia. 
Las columnas siguientes reflejan el precio comercial en UF, la superficie total y el precio en UF por metro cuadrado. Estos datos, irrelevantes para los efectos de este artículo, cobrarán toda su fuerza al momento de cruzar las estimaciones de valor con aquellas de precio.

Cada uno de los indicadores de los parámetros principales está asociado a un valor 1 ó 0 , en función del tipo de externalidad. Si se considera que la incidencia de ese indicador es positiva, entonces se le asignará un valor de 1 como externalidad positiva (Ep). Si su incidencia perjudica la valoración del suelo, se le asignará un valor de 1 como externalidad negativa (En). Esta cifra constituye una información adicional y no incide directamente en el valor final. Su importancia radica en su capacidad de constituirse en una herramienta de ponderación mayor o menor, una vez estimado el valor del suelo.

Las tres columnas siguientes son las más importantes: ponderación, puntaje y acumulado. Estos tres ítemes sintetizan el valor ponderado de ese parámetro en relación al predio total.

La ponderación es la mayor o menor importancia que se le atribuye a un parámetro en relación a otros parámetros y se estima en relación a 1 . Hemos establecido esta ponderación en relación con las tres prioridades antes mencionadas, las que han sido distribuidas de la siguiente manera: los parámetros de primera prioridad tienen una ponderación de 0,085, los de segunda prioridad de 0,034 y los de tercera prioridad de 0,016 . Esta ponderación es un factor que se multiplica por el puntaje o nota.

En este método, el puntaje o nota, según una escala de 1 a 7 , se otorga al parámetro en función del indicador que expresa de la mejor forma la cualidad del terreno. A manera de ilustración, una orientación sur-poniente/nor-oriente con acceso desde el nororiente constituye la orientación preferida por los usuarios dado el régimen de incidencia solar sobre la residencia. Esta orientación recibe un puntaje de 7 . Sin duda que la valoración cambia si se consideran inmuebles de oficinas o educacionales, y cuya orientación mejor evaluada es la oriente- poniente, por cuanto permite una mayor exposición del edificio al sol norte.

El acumulado resulta de multiplicar la ponderación por el puntaje o nota. Así, continuando con nuestro ejemplo, un predio con orientación preferente hacia el nor-oriente, obtendrá una nota 7 la que multiplicada por la ponderación correspondiente, en este caso de 0,085, logra un acumulado de 0,60.

Esta operación se repite para cada uno de los 22 parámetros y, finalmente, se suman todos los acumulados para obtener el valor síntesis del predio. Se trata de una operación matemática muy simple ya que se realiza asociando las tablas de cálculo de aplicaciones del tipo Excel a planos elaborados o disponibles en diversos Sistemas de Información Geográfica (SIG). Cada predio en una aplicación SIG corresponde a un polígono entre cuyos atributos existen tablas a las cuales se asocia la tabla Excel, la que puede ser actualizada cada vez que los datos son modificados. La Tabla de valoración y ponderación de los parámetros ponderados (Cuadro $N^{\circ} 1$ ) se aplica para cada uno de los predios de un loteo proyectado, lo que permite establecer los valores síntesis de todos los predios mediante cifras del 1 al 7 . Estas cifras aparecen en los planos SIG, como lo muestra la Figura $N^{0} 1$.

\section{Estudio de trazados alternativos del territorio}

Finalmente, el valor síntesis, expresado en una cifra de 1 a 7 , da origen a un vector que permite dar una altura al polígono-predio del plano. Este polígono-predio vectorizado constituye la unidad geométrica de lo que denominaremos un manto de valores cualitativos. Este consiste en la construcción de un manto virtual sobre el área a urbanizar, el que expresa gráficamente la mayor o menor valoración que los usuarios otorgan a los diversos predios. Este manto surge de vincular todas las unidades geométricas elaboradas según lo descrito en el título anterior.

Dijimos anteriormente que una de las fortalezas de este método era su capacidad de establecer antes de la proyectación definitiva de un área a urbanizar, los valores estimados de los predios. Es este el momento preciso para establecer esos escenarios apriorísticos. 
Figura $\mathrm{N}^{\circ} 1$

EJEMPLO DE APLICACIÓN DE LA TABLA DE PARÁMETROS EN PREDIOS DE LOMAS DE LO AGUIRRE

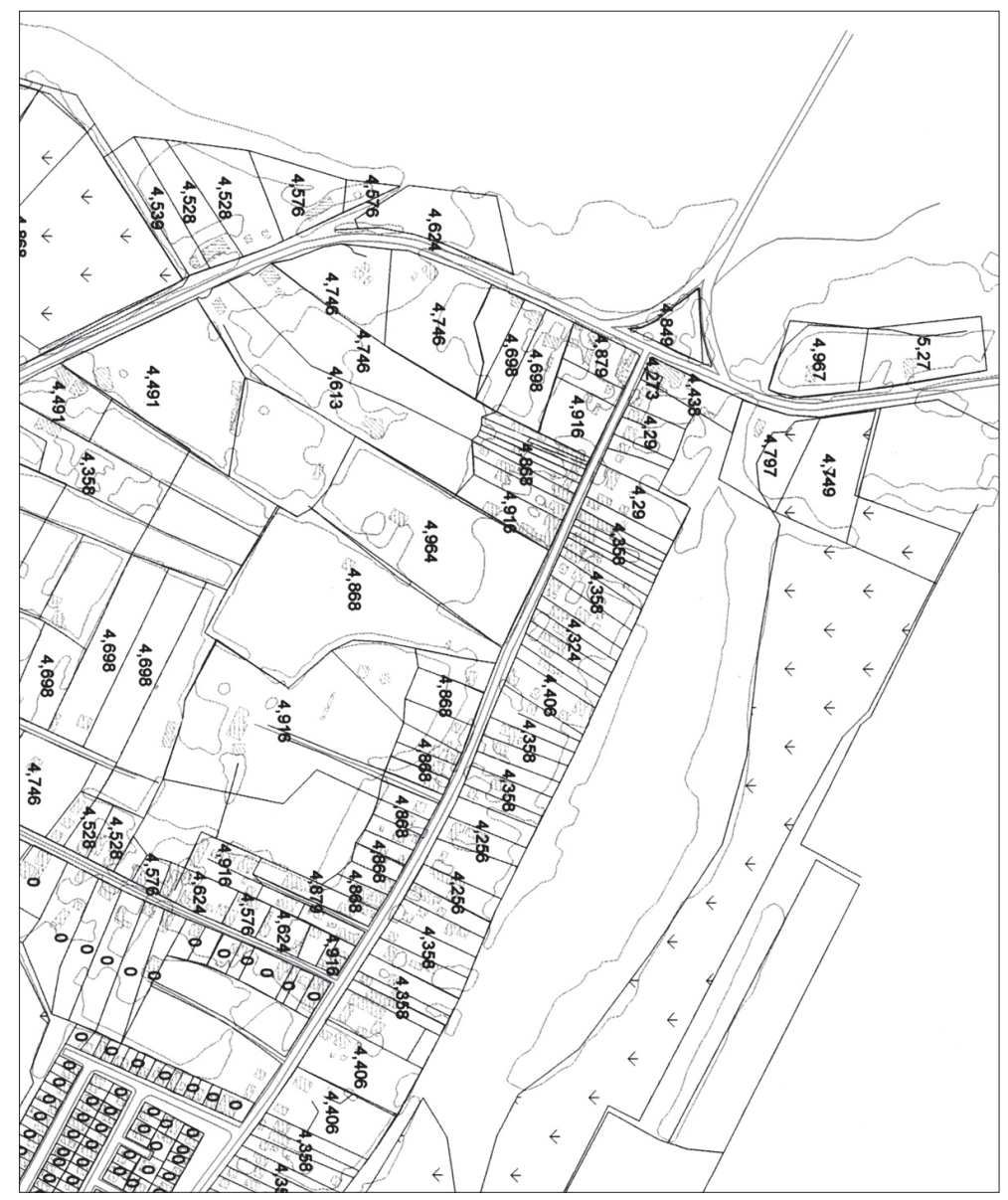

Fuente: Elaboración propia.

Las Figuras $\mathrm{N}^{\circ} 2$ a 5 muestran el caso de un territorio cualquiera, dominado centralmente por una colina. Para este mismo territorio hemos propuesto dos trazados distintos, lo que permite estudiar las diferencias entre los dos mantos de valores cualitativos resultantes. Las Figuras $\mathrm{N}^{\circ} 6$ a $\mathrm{N}^{\circ} 8$ muestran el caso de un mismo trazado para dos territorios distintos.

En el caso del trazado radio-concéntrico (Figuras $\mathrm{N}^{\circ} 2$ y $\mathrm{N}^{\circ}$ 3) los valores aumentan considerablemente hacia la colina, lo que se explica por la excelente combinación entre las posibilidades de adaptación a la pendiente otorgada por las vías concéntricas y las posibilidades de vista, como también el alto nivel de seguridad que implica interiorizarse hacia la colina. En el caso del trazado de damero (Figuras $\mathrm{N}^{\circ} 4$ y $\mathrm{N}^{\circ}$ 5), los valores disminuyen ostensiblemente en el área de la colina y aumentan hacia el perímetro. Esto se explica, entre otras razones, por la excesiva rigidez que implica un damero en pendiente, lo que genera predios con pendientes muy fuertes, vistas deficientes y orientaciones muy dispares entre ellos. 


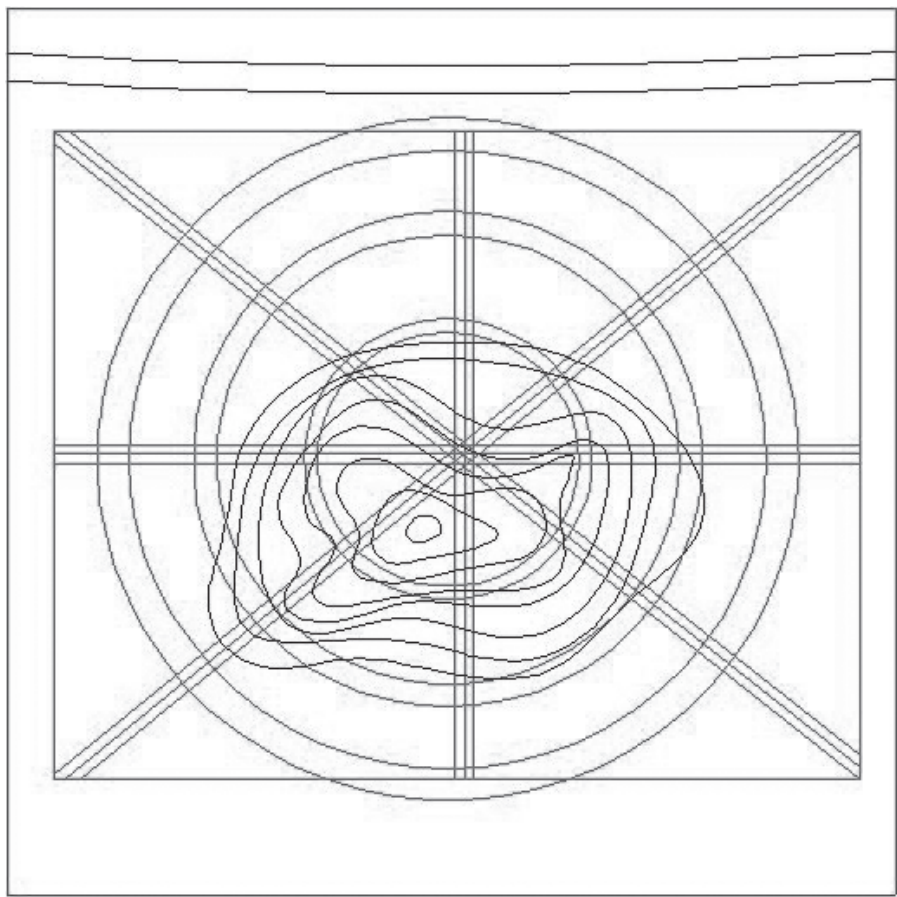

Fuente: Elaboración propia.

Figura $N^{\circ} 3$

ESTUDIO DE TRAZADO 1: MANTO DE VALORES CUALITATIVOS PARA TRAZADO RADIO-CONCÉNTRICO

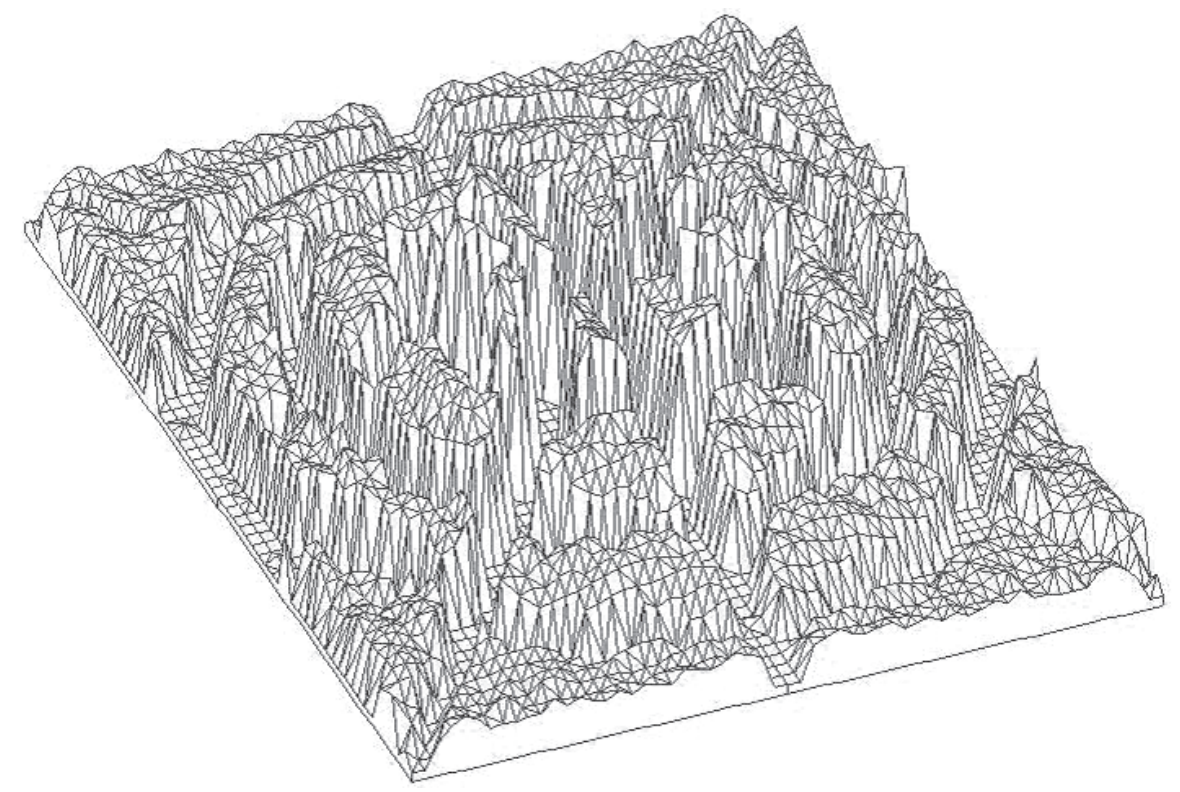

Fuente: Elaboración propia. 
Figura $\mathrm{N}^{\circ} 4$

ESTUDIO DE TRAZADO 2: DE DAMERO EN TERRENO CON COLINA

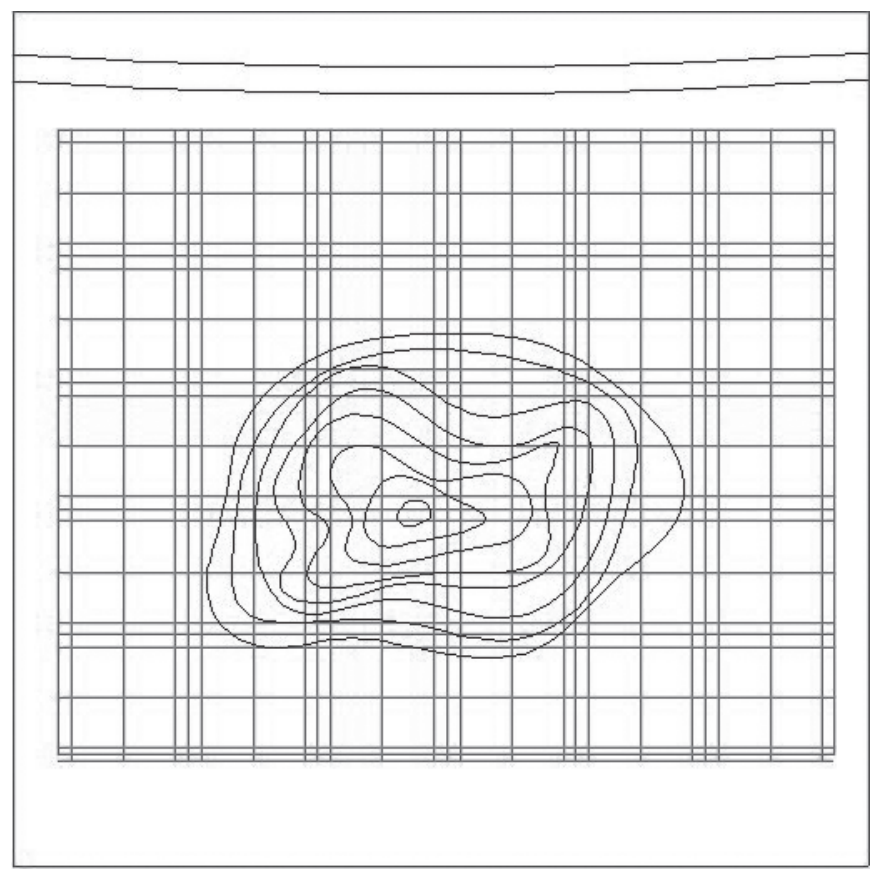

Fuente: Elaboración propia.

Figura $\mathrm{N}^{\circ} 5$

ESTUDIO DE TRAZADO 2: MANTO DE VALORES CUALITATIVOS PARA TRAZADO DE DAMERO

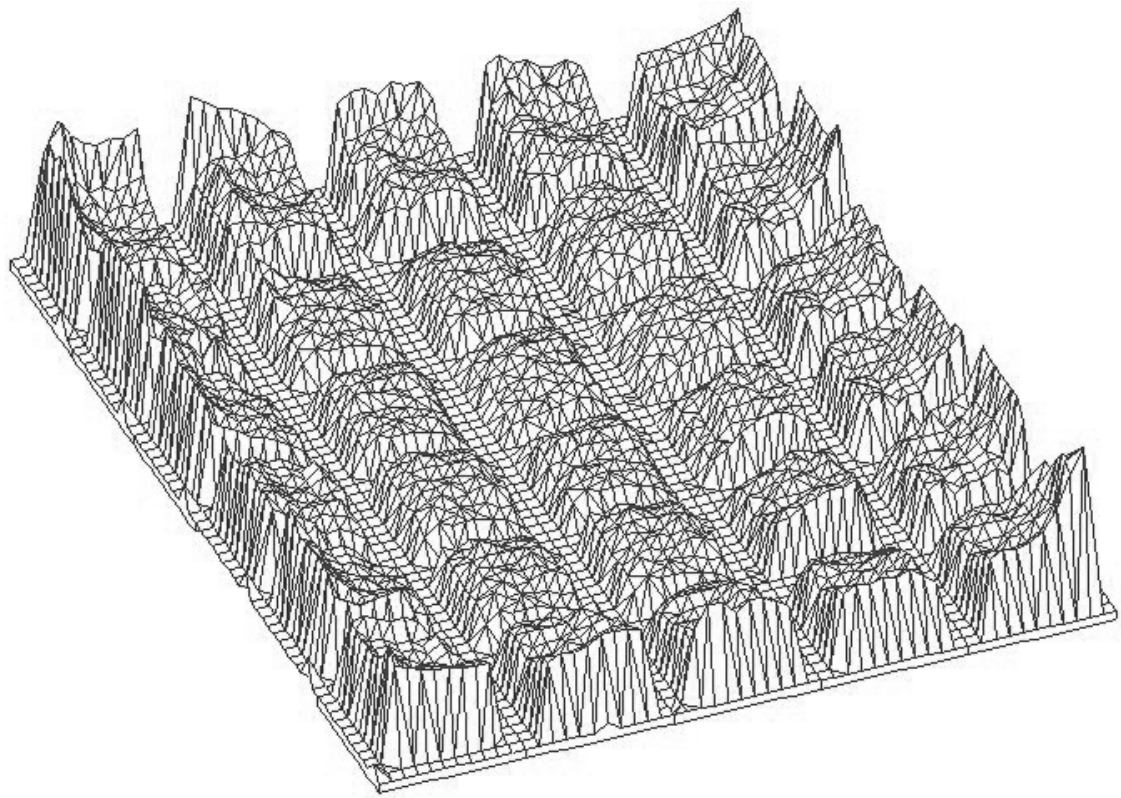

Fuente: Elaboración propia. 
En las Figuras $\mathrm{N}^{\circ} 6$ a 8 las cualidades del territorio cambian radicalmente la valoración del suelo, a pesar que el trazado urbano es el mismo, esto es, un damero regular, atravesado por un río o estero. En la Figura $\mathrm{N}^{0} 7$, el río transporta desechos domiciliarios transformándolo en una suerte de vertedero. No solo es importante la baja valoración de los terrenos adyacentes a este estero sino, además, se puede observar que el conjunto de predios del área a urbanizar disminuyen su valor. En la Figura $\mathrm{N}^{\circ}$ 8, se trata de un río de aguas limpias, aptas para el baño y la recreación. Eso implica una importante valoración de sus predios adyacentes, y la disminución del valor de los predios siguientes se explica por la excesiva diferencia de calidad visual y ambiental que se produce entre los primeros y los segundos.

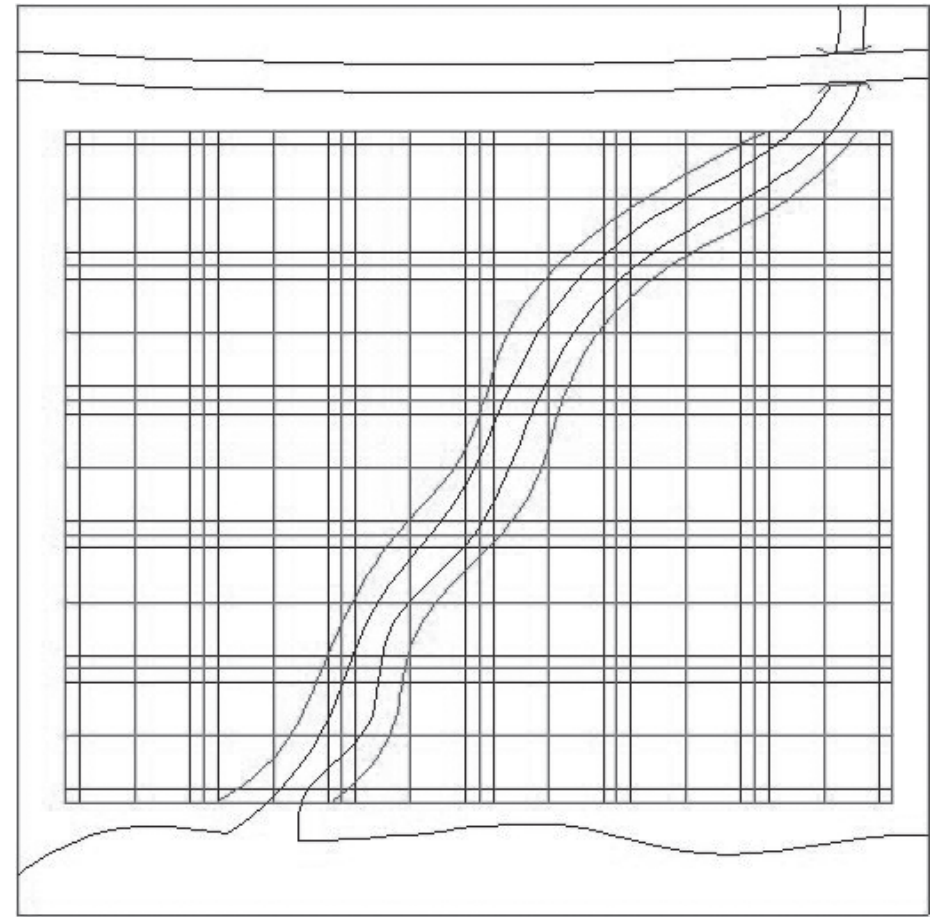

Fuente: Elaboración propia. 
Fuente: Elaboración propia.

Figura $\mathrm{N}^{\circ} 8$

ESTUDIO DEL MISMO TRAZADO 3, CON MODIFICACIÓN DEL TERRITORIO: EL RÍO ES APTO PARA EL BAÑO Y PARA ACTIVIDADES RECREATIVAS

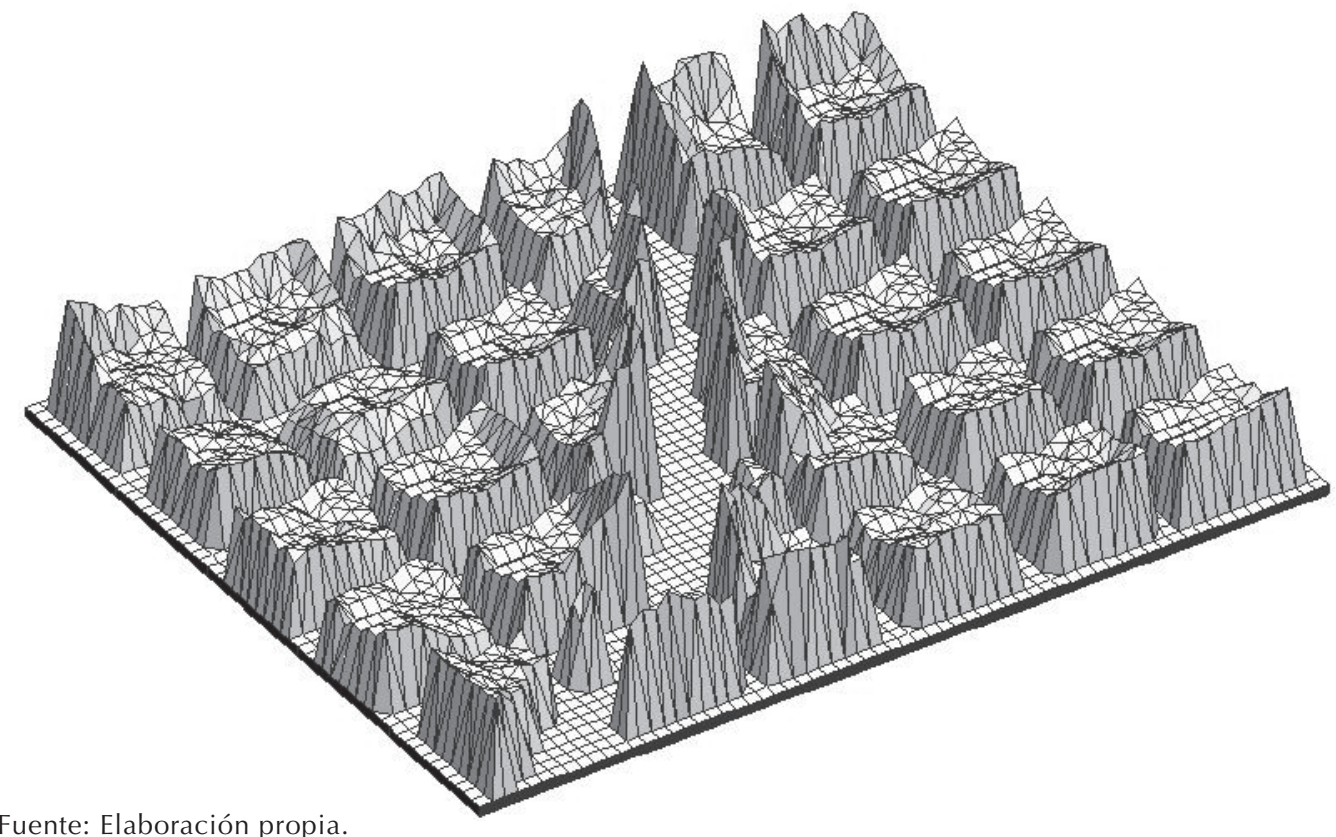

Fuente: Elaboración propia. 
Es interesante notar que en todos los casos mencionados, los parámetros de primera prioridad no tienen gran incidencia sobre la valoración del suelo, incidencia que es muy importante en los parámetros de segunda prioridad. Esto se explica porque los primeros parámetros están implícitos en la decisión de optar por un área a urbanizar: su incidencia se expresa en la decisión de instalarse o no. Una vez tomada esta decisión, los parámetros de segunda prioridad son los más relevantes en la decisión del predio elegido.

\section{Aplicación de la tabla valórica- cualitativa al territorio}

A manera de recapitulación, a continuación se esbozan someramente las grandes etapas de la aplicación del método:

- Se identifican nuevas áreas potenciales a urbanizar a través de un inventario de sitios vacuos, tanto dentro como fuera del área urbanizada. Se estudian sus condiciones legales y territoriales.

- Se identifican actuales y recientes áreas urbanizadas cuyas características geográficas, de localización, de emplazamiento, de tamaño, de orientación y de nivel de ingresos puedan ser asociadas a las nuevas áreas a urbanizar. Se estudia la distribución del precio del suelo con el fin de estimar el estrato económico al que se orienta ese tipo de urbanizaciones. Se estudian además, sobre la base de información entregada por los promotores inmobiliarios y por los usuarios, las principales razones de la fuerte atracción que esas áreas provocan sobre determinados segmentos socioeconómicos de la población.

- La información antes recogida entrega valiosas indicaciones para el desarrollo de los trazados alternativos respecto de: segmento socioeconómico de los usuarios, tamaño y forma de la división predial, tipo de equipamiento y de servicios requeridos, elementos naturales mejor evaluados, formas de accesibilidad, nivel de urbanización de los espacios públicos, tipo de seguridad preferida, etc.
- Lo anterior permite construir una tipología (o cartera) de indicadores mejor evaluados en una urbanización específica, para que por analogías por contraste puedan ser incorporados como variables privilegiadas en los trazados alternativos a desarrollar.

- Se diseñan los trazados alternativos en función de esos indicadores y se aplica la tabla de valoración y ponderación.

- Se levantan los mantos virtuales de valoración.

- Se estudian los diferentes escenarios posibles en función de los objetivos de la urbanización a desarrollar. Se estudian las proyecciones de expansión, la plusvalía posible de los terrenos, las correcciones necesarias a los trazados, etc. Incluso es posible levantar mantos de valoración en los sitios actualmente urbanizados y comparar los resultados con un manto de precios de la misma área. Esto permite observar las variaciones que se producen y establecer las variaciones posibles en las nuevas áreas a urbanizar, a partir de los mantos elaborados.

- Los resultados obtenidos permiten visualizar las modificaciones posibles que se pueden inducir en la orientación natural del crecimiento urbano de la ciudad, en esa zona.

- Se desarrolla el proyecto de loteo definitivo.

\section{Consideraciones finales}

Como lo explica Beaujeu-Garnier, el espacio, los habitantes y el rol de la ciudad, constituyen una trilogía de puntos de vista distintos para entender la ciudad: "Lo que se reúne bajo el nombre de ciudad es multiforme por su posición, por su tamaño, por su arquitectura, por su organización interna, por su rol en la vida regional o nacional" (1997: 11). Uno de esos puntos de vista permite concebir la ciudad como un modo particular de ocupación del suelo, tanto del suelo interior (la ciudad construida) como del suelo exterior (aquella franja 
entre los límites de la ciudad construida y los límites del territorio de influencia de esa ciudad). Una ciudad que ocupa su suelo exterior impacta durablemente las condiciones de vida de su entorno territorial, modificando de paso las cualidades de los asentamientos urbanos menores y rurales que la rodean.

El avance de la ciudad allende sus límites debe mantener las cualidades urbanas de la ciudad que crece, es el primer factor de urbanización del territorio, en el sentido de prolongar, hacia el resto del territorio, la civilización urbana. Consideramos que una ocupación que preserve las cualidades geográficas, sociales, económicas, físico-ambientales del entorno, paisajísticas, de accesibilidad, de la estructura original del suelo y de la forma del territorio puede, por un lado, poner en valor el territorio $y$, por otro, acercar las expectativas de valor que un usuario posee en relación con el precio ofertado del suelo.

Para lograr lo anterior nos ha parecido esencial proponer este método para la valoración económica y cualitativa de un área a urbanizar, de manera de localizar en el espacio los ámbitos periurbanos con mayor probabilidad de demanda de urbanización y con menor impacto territorial. Es una contribución de carácter técnico-metodológico para localizar áreas periurbanas con mayor vocación para ser urbanizadas. Eso permite controlar los modos de ocupación del suelo periurbano y localizar la extensión de la ciudad sobre la base de parámetros explícitos. Ese es el principal aporte de este trabajo a la geografía humana.

A su vez, la originalidad del método consiste en la infinidad de trazados posibles que se pueden modelar y estudiar, frente a un territorio dado, hasta lograr la mayor y más homogénea valoración posible de un área a urbanizar.

De lo anteriormente expuesto es posible deducir cinco aplicaciones y utilidades prácticas de este método:

- Las tipologías e indicadores que se construyen para cada área a urbanizar, en relación a los parámetros de valoración y a las cualidades propias del territorio, permiten identificar fragmentos (Vidal Rojas, 2001) de ciudad que poseen su propia lógica de urbanización y coherencia interna. Extrapolando el método, es posible identificar la organización fragmentaria del conjunto de la ciudad sobre la base de la valoración cualitativa de los suelos urbanizados.

- El método permite establecer límites de carga de los territorios estudiados con el fin de controlar el precio sin disminuir el valor de las propiedades y respetar los valores del territorio.

- El método contribuye a establecer el precio en consideración de los valores asignados de manera de aproximar las expectativas del comprador a las características reales de la urbanización terminada, puesto que el precio reflejará el valor asignado.

- El método es informativo de la mejor inversión a mediano y largo plazo.

- El método es ambiental y económicamente sustentable, en cuanto articula estrategias de preservación del territorio con estrategias de rentabilidad

Respecto de la manera más adecuada de transitar desde este método hacia una aplicación concreta en la ciudad, estimamos necesario generar un plan de intervención urbana que, sobre la base de siete etapas, nos permita pasar de la actual lógica extensiva de crecimiento a un modelo intensivo-desagregado. Esas siete etapas son:

1. Identificación de predios y áreas urbanas y periurbanas no edificadas ni urbanizadas, en la Región Metropolitana;

2. Aplicación experimental del método;

3. Correcciones del método, si se estima necesario;

4. Estudio de la normativa urbana atingente;

5. Valoración de los predios a través del Método de los Valores Tipológicos;

6. Decidir la mejor estrategia y diseño de ocupación del suelo, para una máxima eficiencia construida, con un mínimo impacto territorial; 
7. Elaboración de un Plan de ocupación progresiva de los terrenos periurbanos vacuos localizados dentro del límite urbano, acompañado de un Plan de inversión que ponga en relación los actores públicos y privados, en conformidad con lo establecido en el Plan Regulador Intercomunal Metropolitano (PRIM) de 1994, en el sentido de aumentar la densidad del suelo ya edificado.

\section{Referencias bibliográficas}

ASCHER, F. Los nuevos principios del urbanismo. Madrid: Alianza Editorial, 2004.

BEAUJEU-GARNIER, J. Géographie urbaine. Paris: Armand Colin, 1997.

BIGOT, F. L'urbanisme au défi de I'environnement. Rennes: Ediciones Apogée, Colección Eco Planet, 1994.

BRIDEL, L. Manuel d'aménagement du territoire. Ginebra: Georg Editeur, 1996, Volumen 1.
DERYCKE, P-H. La economía urbana. Madrid: Instituto de Estudios de Administración Local, 1987.

GEDDES, P. Cities in Evolution. Londres: Williams \& Norgate Ltd, 1915.

GRANELLE, J. J. Essai sur la formation des prix du sol dans l'espace urbain. Tesis de Ciencias Sociales. Paris: Universidad de París, 1967.

MARTIN LOU, M. A. y MÚSCAR BENASAYAG, E. Proceso de urbanización en América del Sur. Madrid: MAPFRE, 1992.

REMY, J. et VOYÉ, L. La ville: Vers une nouvelle définition? Paris: L'Harmattan-Entreprises, 1992.

SABATINI, F. Precios del suelo y edificación de viviendas (4 conclusiones sobre Santiago relevantes para políticas urbanas). EURE, 1990, Vol. XVI, N 49, p. 63-72.

VIDAL ROJAS, R. Orientaciones latentes de crecimiento urbano en el Gran Santiago: Constataciones y propuestas. Revista Contribuciones Científicas y Tecnológicas, USACH, 2001, Año XXIX, No 129, p. 53-71. 
